\title{
Estrategias Metacognitivas en los Procesos Creativos. Taller Urbano como Espacio Integrador en la Escuela de Arquitectura de la Universidad del Bío Bío (Concepción, Chile)
}

\author{
Ana Zazo-Moratalla ${ }^{1}$, Carolina A. Arriagada-Sickinger ${ }^{1}$ y Marcela L. Mora-Donoso ${ }^{2}$ \\ (1) Facultad de Arquitectura, Construcción y Diseño, Universidad del Bío Bío, Av. Collao 1202, \\ Concepción, Chile (e-mail: azazo@ubiobio.cl; carriagada@ubiobio.cl) \\ (2) Facultad de Educación y Humanidades, Universidad del Bío Bío, Av. Collao 1202, Concepción, \\ Chile. (e-mail: mamora@ubiobio.cl)
}

Recibido Jun. 28, 2018; Aceptado Sep. 6, 2018; Versión final Sep. 27, 2018, Publicado Abr. 2019

\begin{abstract}
Resumen
El artículo propone realizar una evaluación sobre cómo la metacognición puede colaborar a consolidar la metodología de aprendizaje basado en problemas y pretende arrojar luz sobre la importancia su incorporación en los procesos de enseñanza de la arquitectura y de los procesos creativos. Se exponen los resultados de la aplicación del análisis metacognitivo en la asignatura de Taller V de Diseño Urbano, impartida el último año lectivo de la carrera de Arquitectura en la Universidad del Bío Bío. Esta asignatura integra los conocimientos previos adquiridos en las asignaturas teóricas de Urbanismo I, II y III y en los niveles prácticos de Taller I a IV, mediante el método de aprendizaje basado en problemas. Para ello se utilizó el instrumento de aprendizajes previos (Knowledge and Prior Study Inventory) y el grupo focal. Los resultados demuestran que su uso generó resultados positivos permitiendo realizar una toma de conciencia de los conocimientos abstractos adquiridas.
\end{abstract}

\section{Metacognitive Strategies in Creative Processes. Urban Workshop as an Integrated Space in the Architectural School of the Bio Bio University (Concepción, Chile).}

\begin{abstract}
This article proposes an evaluation of how metacognition can help to consolidate the problem-based learning methodology and shed light on the importance of its incorporation into the processes of teaching architecture and creative processes. The results of the application of the metacognitive analysis in Taller $V$ (Workshops of Urban Design) are exposed. This subject is taught during the last academic year of the Architecture Career at the University of Bío Bío. Taller $V$ integrates the previous knowledge acquired in the theoretical subjects of Urbanism I, II y III and the practical subjects of Taller I to IV by means of the method of learning based on problems. The Knowledge and Prior Study Inventory instrument and the focus group were used. The results show that the use of this tools generated positive outcomes allowing to become aware of the abstract knowledge acquired along the creative process.
\end{abstract}

Keywords: didactic project; learning; creative process; urban design; metacognition 


\section{INTRODUCCIÓN}

La literatura internacional relativa a la enseñanza universitaria de la arquitectura es actualmente un tema candente (Mutaqi, 2018; Sawyer, 2018). Los estudios de John Dewey sobre el aprendizaje activo (Dewey el al., 1951) en la escuela de la Bauhaus durante la década de los 40 y de Donald Schön sobre la reflexiónacción (Schön, 1995) en las décadas de los 80 y 90, marcaron las bases de la didáctica en arquitectura. En la actualidad, diversas corrientes utilizan estos métodos para enfocar la pedagogía proyectual y el proceso creativo sobre diversos aspectos como el diseño de una arquitectura y un entorno urbano más sostenible y resiliente (Bassanese, 2018; Donovan, 2018), la investigación (Muñoz, 2009), la morfogénesis (Voyatzaki y Gourdoukis, 2018) o el uso de tecnologías emergentes (Gómez-Galán, 2018). Sin embargo, los análisis enfocados en los procesos de aprendizaje de los alumnos de arquitectura en el aula y, particularmente, la metacognición (Nelson, 2002) en los procesos creativos son menos frecuentes (Abusaada y Elshater, 2018).

El presente artículo expone los resultados de una estrategia de innovación pedagógica basada en indicadores de desempeño metacognitivos e implementada durante el curso 2017 en la asignatura de Taller $V$ de Diseño Urbano de la carrera de Arquitectura en la Universidad del Bío Bío (en adelante UBB), ubicada en la ciudad de Concepción Chile. Esta asignatura se imparte durante el último nivel en la carrera antes de dar paso al proyecto de título que otorga la licenciatura a los estudiantes de arquitectura. En ella, el alumno da el salto al diseño urbano oscilando entre las escalas de trabajo de ciudad, barrio y espacio público. De este modo, el alumno cierra su proceso académico con una visión integradora del papel del arquitecto, incorporando las estrategias que le permitirán desenvolverse a esa escala de trabajo. En este nivel se plantea el desafío de integrar todos los conocimientos previos adquiridos mediante un método de aprendizaje basado en problemas, de modo que, a través de una metodología de trabajo ya consolidada en la UBB, los alumnos sean capaces de aplicar las diversas herramientas a través de las diferentes escalas de trabajo y consigan desarrollar un diseño urbano adecuado a la problemática propuesta en el aula.

La relevancia del ejercicio de metacognición propuesto es que esta asignatura actúa de espacio integrador de dos líneas de trabajo desarrolladas durante los cuatro años previos de la carrera de Arquitectura. Una derivada de un proceso creativo arquitectónico y otra que incorpora las técnicas de análisis e intervención urbana aprendidas en las asignaturas teórico-prácticas de Urbanismo I, II y III, según se indica en la siguiente figura $\mathrm{N}^{\circ} 1$, en las que se trabaja una dimensión propositivo-práctica a las escalas manzana, barrio y ciudadmetrópolis respectivamente.

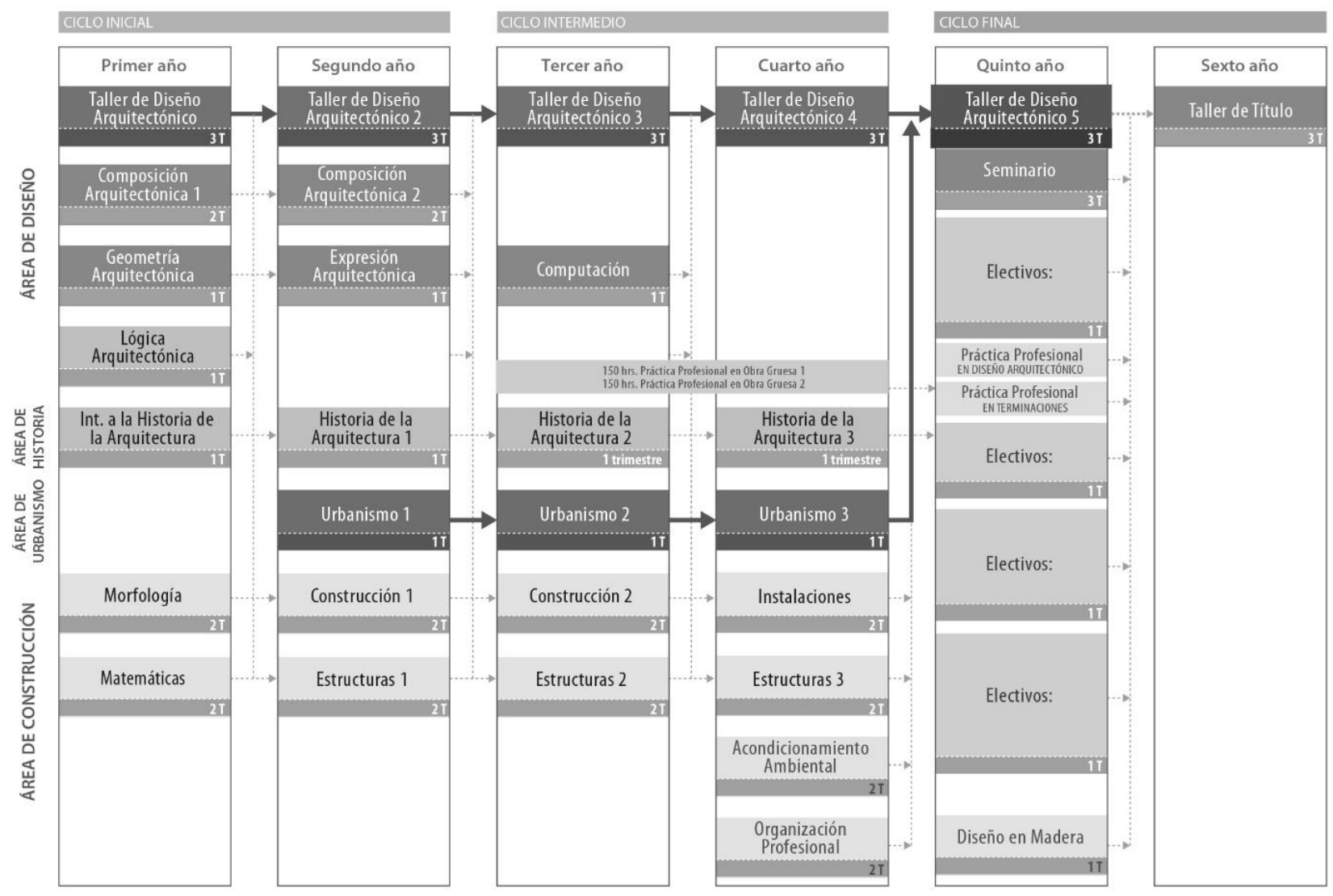

Fig. 1: Ubicación de la asignatura en el plan de estudios anual de la Carrera de Arquitectura de la UBB. 
El objetivo concreto es el de evaluar si el uso de instrumentos metacognitivos complementando a la metodología de trabajo asentada en Taller $V$ de Diseño Urbano consolida el proceso de aprendizaje de los alumnos. Se parte de la hipótesis de que la metodología aplicada en el aula, apoyada en los instrumentos de metacognición, permite afianzar los saberes y las herramientas proyectuales que los propios alumnos construyen en el proceso creativo. Como objetivo de largo alcance, este caso de estudio trata de arrojar luz sobre cómo la metacognición puede ayudar a consolidar la metodología de aprendizaje basado en problemas y de la importancia su incorporación en los procesos de enseñanza de la arquitectura y de los procesos creativos.

El artículo se organiza en seis apartados. En el primero, se realiza un breve repaso sobre los métodos la didáctica proyectual en taller en las escuelas de arquitectura. En el segundo, se expone el instrumento aplicado para desarrollar el ejercicio en el aula, el Knowledge and Prior Study Inventory (en adelante KPSI). Posteriormente, se realiza la descripción metodológica de la implementación de la innovación pedagógica en el aula. En el siguiente apartado se describen los resultados de la aplicación de los instrumentos de metacognición, siendo discutidos posteriormente. Finalmente se concluye cuestionando el grado de relevancia que tiene la metodología aplicada y se argumenta en función de las opiniones y saberes de los alumnos.

\section{LA METACOGNICIÓN EN LOS PROCESOS CREATIVOS}

Según Mabardi (2012:82) "la arquitectura [es] un orden que cualifica, por medio de un artefacto, el espacio del ser y del devenir de un grupo de seres humanos con un contexto geográfico y cultural o multicultural", siendo el proyecto el resultado creativo. Su enseñanza, por tanto, no sólo debiera estar relacionada con el edificio como artefacto o el diseño urbano como resultado, sino también con las operaciones cognitivas figurativas que le dan forma (Lagos, 2005). A su vez, (Cross, 1990) describe el proceso de diseño como exploratorio, teórico, emergente, oportunista, reflexivo y de esfuerzo arriesgado, debiendo las instituciones que imparten su educación desarrollar dichas características en los procesos de aprendizaje. Un punto de partida para este aprendizaje es el proceso cognitivo que los estudiantes desarrollan en resolver un proyecto, o problema, de forma natural. Hargrove (2013) refuerza esta idea señalando que la forma de enseñanza tradicional necesita desesperadamente el soporte de las habilidades metacognitivas, como la capacidad de autorregular los procesos de aprendizaje, para mejorar tanto las competencias teóricas como las prácticas en el proceso creativo.

\section{La didáctica en Arquitectura: el taller arquitectónico-urbano}

Tradicionalmente la didáctica del Taller de Arquitectura se ha centrado en métodos de aprendizaje basado en problemas, aplicados al objeto arquitectónico y al contexto urbano. Este método, desarrollado en los años 70 por un grupo de educadores médicos de la Universidad de Mc Master (Canadá), se caracteriza por estar basado en el estudiante, por promover un aprendizaje significativo y por desarrollar las habilidades y competencias necesarias para el contexto profesional (Morales y Landa, 2004) definidas por las mallas curriculares (Icarte y Labate, 2016). Según Raposo, (2014, p.97) "la artística está ligada a la resolución de determinados problemas, que empiezan por esa situación abierta, en la que sirven todos los caminos, y que posteriormente se van acotando". Con este método, el profesor "enseña" en Taller en la etapa inicial de diseño construyendo una experiencia educacional que afecta la manera de pensar de los alumnos y de trabajar en el proceso creativo (Atman et al., 2005) para, posteriormente, enfrentar al alumno con la resolución de un problema y actuar como orientador. El proceso creativo, bajo este método, promueve ir más allá de las ideas del presente, lidiando en un entorno social y ambiental complejo, enfrentando al alumno con el mundo real (Hargrove, 2013), alentado a un efectivo, productivo y funcional modo de pensar (Uzunoglu y Uzunoglu, 2011) y otorgándole las herramientas necesarias para enfrentar cualquier desafío.

Además, la didáctica del taller de arquitectura se enmarca el paradigma constructivista (Coll et al., 1997), donde el alumno es el constructor de su propia estructura de conocimiento. Este enfoque en el contexto arquitectónico también ha sido denominado teoría de la integración del conocimiento (Salama, 2008) mediante el cual el alumno va incorporando en su acervo conocimientos aprendizajes complementarios y que funcionan de forma sistémica. En este marco el profesor se enfoca en la construcción del conocimiento a través de actividades auténticas basadas en las experiencias ricas en contexto (Hernández, 2008). La metodología de enseñanza del taller de Diseño Urbano integra un proceso creativo de desarrollo práctico-propositivo y otro teórico de técnicas de análisis y diagnóstico urbano. El objetivo de esta escala supera la del objeto arquitectónico generando una visión crítica de los estudiantes frente a los modelos de desarrollo urbano vigentes (Arze et al., 2012). 


\section{La evaluación metacognitiva: el KPSI}

En el actual contexto de la formación universitaria se hace indispensable implantar la evaluación metacognitiva sobre los aprendizajes adquiridos en los procesos de aprendizaje, tanto metódicos como creativos. El énfasis de este tipo de evaluación es el de generar la reflexión desde su propia práctica y formar alumnos autónomos, sobre la base de una educación que potencie la conciencia sobre los propios procesos cognitivos y la autorregulación de los mismos por parte de los estudiantes (Osses y Jaramillo, 2008). Lo esencial es que en la formación profesional se entregue las herramientas a los estudiantes para que apliquen las habilidades de pensamiento ejecutivas ((Diamond y Lee, 2011), entendiendo como tal la planificación, el control o la supervisión y la evaluación de lo realizado.

En este escenario, la metacognición según Glaser (1994), es una de las áreas de investigación que más ha contribuido a la configuración de las nuevas concepciones del aprendizaje. Su pionero fue Flavell (1976: 232), quien utilizó este término para afirmar que la metacognición hace referencia, por una parte, "al conocimiento que uno tiene acerca de los propios procesos y productos cognitivos o cualquier otro asunto relacionado con ellos", y, por otro, "a la supervisión activa y consecuente regulación y organización de estos procesos" (Osses y Jaramillo, 2008, p.191). El grado de comprobación de los conocimientos previos por parte del estudiante fue abordado por un instrumento evaluativo metacognitivo: el Knowledge and Prior Study Inventory o KPSI (Young y Tamir, 1977) adaptando una propuesta previa de Lazo (2012), quien utilizó un instrumento metacognitivo para la enseñanza de la Química. Lazo diseñó afirmaciones relativas a un contenido con el objetivo de que los estudiantes generaran su propia "toma de conciencia" en relación al nivel de conocimiento sobre conceptos claves para abordar una temática nueva en ciencias.

En esta investigación se utiliza la propuesta que realiza Burón (1990) de dimensión del entrenamiento metacognitivo o en el control, en la que el proceso metacognitivo avanza respecto de la instrucción razonada. En ella, el profesor, además de explicar a los alumnos la utilidad de usar una estrategia concreta, los orienta a que ellos lo comprueben y así, indirectamente, aporta en la toma de conciencia de su efectividad.

\section{Descripción metodológica de la Implementación de innovación pedagógica}

Durante el curso 2017 la sección 3 del plan anual de la carrea de Arquitectura de la Universidad del Bío Bío propone como ejercicio trabajar el borde costero en Penco (Figura 2). El gran desafío propuesto es el de integrar la trama urbana al borde costero asegurando condiciones de habitabilidad, sustentabilidad y seguridad. El trabajo se divide en dos etapas. La primera, desarrollada de forma grupal (6 grupos de 4 alumnos) en la que se trabaja a escala urbana realizando un análisis, un diagnóstico urbano y una propuesta de plan maestro estratégico bajo una idea fuerza o imagen objetivo desentrañada del diagnóstico. La segunda, desarrollada de forma individual, en la que se trabaja a escala barrial precipitando el diagnóstico y las propuestas desarrolladas en la etapa anterior de forma que se proponga una ordenación del barrio y una aproximación al diseño urbano del área.

El objetivo de esta metodología es el de analizar el aprendizaje de los alumnos de Taller V mediante la activación de un proceso de metacognición individual sobre los conocimientos conceptuales aplicados y sobre los conocimientos procedimentales y actitudinales adquiridos durante el proceso del proyecto propuesto. Para ello, se propone aplicar dos técnicas de evaluación, una individual durante el proceso y otra grupal al final del proceso. La primera tiene un carácter más formativo, ya que tiene como objetivo determinar las dificultades de cada alumno/a en su proceso de aprendizaje durante el proceso de trabajo. La segunda tiene un carácter más sumativo ya que tiene como objetivo establecer balances fiables de los resultados obtenidos al final del proceso.

Para el primer tipo de evaluación se propone como instrumento el uso del formulario KPSI al final de cada parte del proceso del proyecto (análisis y diagnóstico grupal, plan maestro grupal y plan maestro individual) con el objetivo de diseccionar cada una de los elementos que constituyen estas fases y evaluar de forma individual las dificultades encontradas en la aplicación de los diversos conceptos, en la integración de los mismos, en la transformación y en su representación. El diseño de los tres instrumentos aplicados se realiza de forma específica para cada parte del proceso que poseen una metodología en sí mismas: análisis y diagnóstico grupal, plan maestro grupal y plan maestro individual. Para cada una de ellas se determinan tres tipos de preguntas orientadas a evaluar diferentes tipos de dificultades, (A) dificultades en el entendimiento de conceptos, (B) dificultades en el desarrollo del proceso y (C) dificultades en representación y comunicación de sus ideas. La ordenación de las preguntas se realiza de forma que tienen sentido con el proceso aplicado más que por bloques de evaluaciones. De este modo, las preguntas de los KPSI quedaron ordenadas de la siguiente forma que se indica en las tablas 1,2 y 3 : 


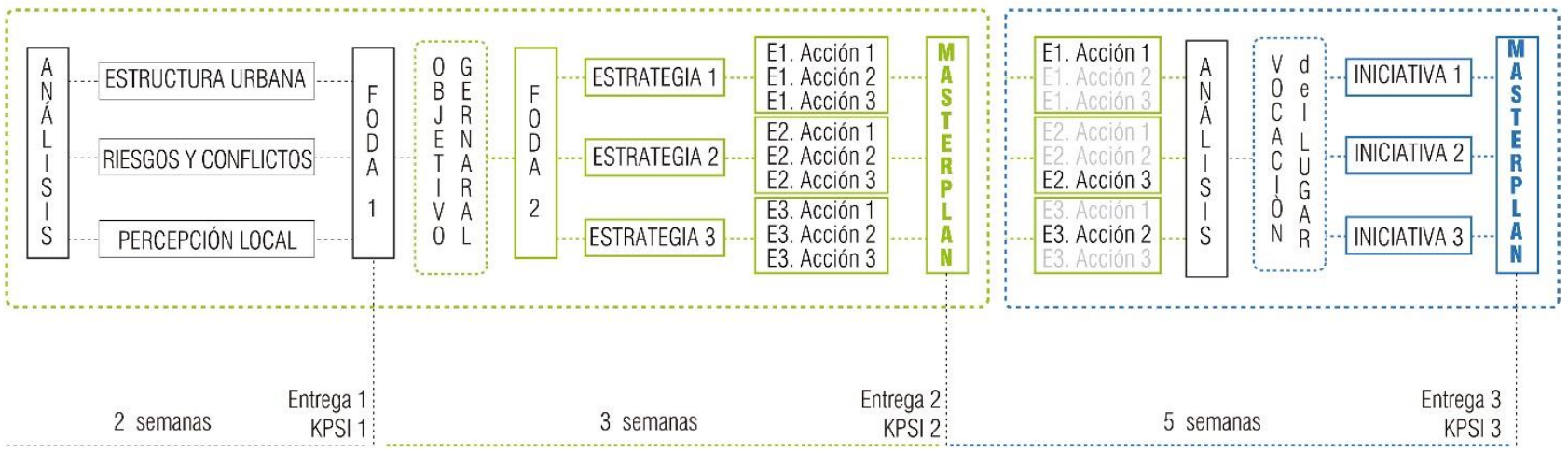

Fig. 2: Diagrama metodológico con indicación de los momentos de aplicación del KPSI.

Tabla 1: KPSI aplicado tras la primera fase de análisis y diagnóstico grupal

\begin{tabular}{|l|l|l|l|}
\hline Preguntas & A & B & C \\
\hline 1) Identifico los elementos de un análisis espacial & & X & \\
\hline 2) Identifico los elementos de un análisis funcional & & X & \\
\hline 3) Soy capaz de discernir entre conflictos y riesgos & $\mathrm{X}$ & & \\
\hline 4) Soy capaz de discernir entre problemas ambientales y antrópicos & $\mathrm{X}$ & & \\
\hline 5) Identifico hitos, nodos, distritos, bordes y sendas. & & $\mathrm{X}$ & \\
\hline $\begin{array}{l}\text { 6) Soy capaz de diferenciar las fortalezas, de las oportunidades, de las amenazas } \\
\text { y de las debilidades. }\end{array}$ & $\mathrm{X}$ & & \\
\hline 7) Soy capaz de jerarquizar la información en base a un criterio seleccionado & & $\mathrm{X}$ & \\
\hline 8) Entiendo el proceso global del diagnóstico urbano & & $\mathrm{X}$ & \\
\hline 9) Soy capaz de graficar todas las ideas que propongo & & $\mathrm{X}$ \\
\hline
\end{tabular}

Tabla 2: KPSI aplicado tras la segunda fase de plan maestro grupal

\begin{tabular}{|l|l|l|l|}
\hline Preguntas & A & B & C \\
\hline $\begin{array}{l}\text { 1) Soy capaz de jerarquizar la información del FODA en base a un criterio } \\
\text { seleccionado }\end{array}$ & X & \\
\hline 2) Entiendo la diferencia entre estrategia y acción & $\mathrm{X}$ & & \\
\hline 3) Soy capaz de traducir las oportunidades y amenazas en estrategias & & $\mathrm{X}$ & \\
\hline 4) Soy capaz de relacionar las estrategias con las acciones & & $\mathrm{X}$ & \\
\hline 5) Entiendo cómo se definen las acciones & & $\mathrm{X}$ & \\
\hline 6) Soy capaz de graficar todas las ideas que propongo en términos estratégicos & & & $\mathrm{X}$ \\
\hline 7) Entiendo el proceso global del máster plan estratégico & & $\mathrm{X}$ & \\
\hline
\end{tabular}

Tabla 3: KPSI aplicado tras la tercera fase de plan maestro individual

\begin{tabular}{|l|l|l|l|}
\hline Preguntas & A & B & C \\
\hline $\begin{array}{l}\text { 1) Soy capaz de formular una vocación del lugar coherente con el objetivo general } \\
\text { del plan maestro }\end{array}$ & $\mathrm{X}$ & \\
\hline 2) Soy capaz de traducir la vocación en un esquema conceptual & & $\mathrm{X}$ & \\
\hline 3) Entiendo la diferencia entre acción e iniciativa & $\mathrm{X}$ & & \\
\hline 4) Soy capaz de traducir las acciones en iniciativas & & $\mathrm{X}$ & \\
\hline 5) Soy capaz de espacializar las iniciativas & & $\mathrm{X}$ & \\
\hline 6) Soy capaz de graficar todas las ideas que propongo en términos espaciales & & & $\mathrm{X}$ \\
\hline $\begin{array}{l}\text { 7) Entiendo el proceso de plan maestro (desde las acciones hasta la } \\
\text { espacialización de las iniciativas) }\end{array}$ & $\mathrm{X}$ & \\
\hline
\end{tabular}


Tras proporcionarles el cuestionario a los alumnos se les solicita que realicen una metaevaluación de sus aprendizajes y dificultades de forma que respondan con honestidad (no anónimo) según la siguiente graduación: 1) No lo sé / No lo comprendo; 2) Lo conozco un poco; 3) Lo comprendo parcialmente; 4) Lo comprendo bien; y 5) Lo puedo explicar a un compañero.

Para el segundo tipo de evaluación en la investigación se propone como técnica el desarrollo de un grupo focal (o grupo de conversación). Se trata de una técnica cualitativa para la cual se diseña un instrumento conformado que analiza el avance de los conocimientos a través del desarrollo del proyecto, en sus tres etapas. Su objetivo es evidenciar conceptualizaciones claves, procedimientos, metodologías utilizadas y actitudes manifestadas en el desempeño del proyecto en el marco del taller de arquitectura. Las preguntas del grupo focal se organizaron en tres ámbitos. El primero, conocimientos conceptuales: aquellos conceptos teóricos claves que permitieron desarrollar el proyecto. El segundo, conocimientos procedimentales: aquellos procedimientos, metodologías, paso a paso para la concreción del proyecto. El tercero, conocimientos actitudinales: aquellas actitudes profesionales que deben estar presentes en el desarrollo de cualquier proyecto: tales como: rigor científico, aplicación de normas éticas, aplicación de la normativa pública, orientación al trabajo en equipo.

\section{Resultados de la aplicación de los instrumentos de metacognición en Taller $V$}

Se analiza en primer lugar los resultados por separado de cada instrumento de conocimientos previos por separado y del grupo focal final. En los siguientes apartados de discusiones y conclusiones se revisan los resultados de forma transversal y se pone en relación con la problemática presentada en la introducción vinculada a la metacognición en los procesos creativos.

El primer instrumento de conocimientos previos (KPSI) fue construido con nueve enunciados que hacen referencia a los conceptos claves de la primera etapa del proyecto y se aplicó tras la primera fase de análisis y diagnóstico urbano. Este instrumento. Los resultados obtenidos muestras que, de los 24 estudiantes participantes, un $49 \%$ está en el nivel de logro 4, que equivale a: lo comprendo bien; un $27 \%$ está en el nivel de logro 3, lo comprendo parcialmente; un $22 \%$ está al nivel de logro 5, lo puedo explicar a un compañero, mientras que sólo un $2 \%$ manifiesta la opción 2, que hace referencia al nivel de comprensión: lo conozco un poco y no se observa preferencias en el nivel 1, referente a: no lo sé, no lo comprendo. Este primer escenario de respuestas en relación a un proceso metacognitivo, en que el estudiante somete a juicio respecto a ciertos enunciados que se presentan en el instrumento, permite abordar con mayor precisión aquellos conceptos claves que deben ser reforzados antes de comenzar la tarea de proyecto, con el fin de llegar a resultados positivos del punto de vista del aprendizaje.

El segundo instrumento fue construido con siete enunciados que hacen referencia a conceptualizaciones básicas y procedimientos para abordar una propuesta estratégica urbana derivada del análisis y diagnóstico previo y que se aplicó tras el cierre del Master Plan Estratégico. Los resultados obtenidos muestran que la tasa de respuesta de mayor frecuencia está en el nivel de logro 4, lo comprendo bien, con un $54 \%$, posteriormente se observa en nivel 3 , lo comprendo parcialmente, con un $23 \%$, en un nivel 5 , Lo puedo explicar a un compañero con un $21 \%$ y con un $2 \%$ nivel 2 , lo conozco un poco. No se observa tasa de respuesta en nivel 1 , no los sé, no lo comprendo.

El tercer instrumento fue construido con siete enunciados que hacen referencia a la capacidad de transformar las estrategias en acciones concretas de diseño urbano y que se aplicó tras el cierre del Máster Plan Individual. Se observa que todos los niveles tienen tasa de respuesta, situación que no se presentan en las aplicaciones de la etapa 1 y 2 . Los resultados muestran un $42 \%$ en el nivel 3, Lo comprendo parcialmente, un $37 \%$ en el nivel 4 , lo comprendo bien, y un $8 \%$ en el nivel 1 , No lo sé, no lo comprendo y un $2 \%$ en el nivel 5 , lo puedo explicar a un compañero.

Por otro lado, la técnica del grupo focal se aborda desde la formulación de categorías que permite analizar los discursos vertidos por parte de los estudiantes que participaron en la técnica, y que a su vez estuvieron presente en el proceso de aprendizaje en el taller. Se aplicó en el análisis una técnica de análisis de contenido, levantando categorías previas sustentadas en los tres ejes: conocimientos conceptuales, procedimentales y actitudinales, y a su vez, aquellos conocimientos que todo/a arquitecto/a debe manifestar. Para visualizar los resultados se diseñó la siguiente tabla 4, que contiene fragmentos claves de las intervenciones, y síntesis interpretativas de ellas, según los ejes antes señalados.

\section{DISCUSIÓN}

En una primera instancia, y como lectura transversal de los resultados se detectó que los alumnos no están acostumbrados al ejercicio metacognitivo. En el desarrollo de la asignatura realizan una tarea sin evidenciar 
o hacer consciente las herramientas que están utilizando, los conceptos o procedimientos que les resulta más complicado incorporar a su acervo, o cuáles son las partes para las que llegaban más preparados o les resultaba más fácil acometer. A su vez se puede establecer que, a nivel general, las tendencias de las respuestas de los instrumentos de conocimientos previos (KPSI) están en el nivel 3 (Lo comprendo parcialmente) y 4 (lo comprendo bien).

Tabla 4: Síntesis

\begin{tabular}{|c|c|c|}
\hline Ejes de Análisis & Fragmentos claves & $\begin{array}{l}\text { Síntesis interpretativa, } \\
\text { conceptualizaciones } \\
\text { relevantes }\end{array}$ \\
\hline \multirow[t]{6}{*}{ Conceptual } & $\begin{array}{l}\text { Fragmento 1: "se parte es como por la sensibilidad del contexto, lo } \\
\text { que en realidad es entender que es un contexto que está } \\
\text { compuesto por varias dimensiones y, o sea, y varias de ellas } \\
\text { materiales y otras que son etéreas". }\end{array}$ & $\begin{array}{l}\text { Sensibilidad del contexto, } \\
\text { variables del contexto, } \\
\text { entorno construido. }\end{array}$ \\
\hline & $\begin{array}{l}\text { Fragmento } 2 \text { "La historia está detrás de lo que generó ese entorno } \\
\text { construido" }\end{array}$ & \multirow{3}{*}{$\begin{array}{l}\text { Taxonomía para } \\
\text { jerarquizar. } \\
\text { Diseño desde tres } \\
\text { perspectivas: desde la } \\
\text { visión del arquitecto, desde } \\
\text { las personas, del entorno. } \\
\text { Defender las posturas a } \\
\text { partir de enfoques. }\end{array}$} \\
\hline & $\begin{array}{l}\text { Fragmento } 3 \text { "El medio ambiente está primero como contexto y si } \\
\text { está muy urbanizado hay que hacer una taxonomía que es todo lo } \\
\text { que componen ese entorno construido tan complejo". }\end{array}$ & \\
\hline & $\begin{array}{l}\text { Fragmento 4: "cuando llegamos al taller urbano nosotros } \\
\text { empezamos a eh desarrollar o entender la... la responsabilidad del } \\
\text { arquitecto en la ciudad y en eso hay como dos caminos súper } \\
\text { claros por el cual tomar: si es que yo diseño a partir de lo que yo } \\
\text { como arquitecto o como grupo de arquitectos creemos a partir } \\
\text { como academia lo que es bueno para la ciudad eh... o la segunda } \\
\text { que es diseñar para las personas y otra tercera que es... ya } \\
\text { empieza a mezclar las dos cosas que es yo creo que la ideal y } \\
\text { trabajando en grupo, que eso ya lo hemos visto en los urbanismos } \\
\text { anteriores eeh, empiezan a luchar esa... esas posiciones" }\end{array}$ & \\
\hline & $\begin{array}{l}\text { Fragmento 5: "Entonces ese ese tipo de posturas que empezamos } \\
\text { a tomar en los años anteriores son las que se empiezan a reflejar y } \\
\text { las que se empiezan a discutir este año. Creo que por ese lado } \\
\text { eem... es interesante de entender eso... cada uno de esos } \\
\text { conceptos porque tiene mucho que ver con la... con la postura del } \\
\text { arquitecto frente a la ciudad y frente al proyecto" }\end{array}$ & \multirow[t]{2}{*}{$\begin{array}{l}\text { Formular síntesis es lo más } \\
\text { clave en este taller }\end{array}$} \\
\hline & $\begin{array}{l}\text { Fragmento 6: "yo creo que eh una de las cosas importantes dentro } \\
\text { del primer ejercicio fue como hacer el hincapié en que pudiésemos } \\
\text { llegar a hacer como una síntesis, como a sintetizar la información y } \\
\text { poder darle una línea y una carácter, porque... o sea yo creo que al } \\
\text { momento de intervenir si bien hay que entender todas estas } \\
\text { dimensiones" }\end{array}$ & \\
\hline \multirow[t]{2}{*}{ Procedimental } & $\begin{array}{l}\text { Párrafo 7: "que era esto de las iniciativas, o sea, porque si se } \\
\text { entendía cuando los explicaban, pero después al llevarlo a cuando } \\
\text { teníamos que trabajarlo en el lugar eh... como que las cosas se } \\
\text { empezaban a confundir, empezábamos a pensar en que eran las } \\
\text { oportunidades, en que era esto" }\end{array}$ & \multirow{2}{*}{$\begin{array}{l}\text { En talleres anteriores } \\
\text { pasábamos a la acción, aquí } \\
\text { observamos etapas, las } \\
\text { correcciones permitieron } \\
\text { clarificar. } \\
\text { Aplicación del análisis } \\
\text { urbano de Kevin Lynch }\end{array}$} \\
\hline & $\begin{array}{l}\text { Párrafo 8: "llevábamos mucho tiempo acostumbrados como a pasar } \\
\text { muy directos a la acción, entonces como que todo lo que veíamos } \\
\text { lo pensábamos al tiro en como queríamos que fuese o como } \\
\text { imaginábamos como si iba a ver si como todas estas... esta serie } \\
\text { de capas de... de cómo... de... digamos eh... decantamos la } \\
\text { información hasta un... Y siento que no sé, como que a mi parecer } \\
\text { nos pasó a varios que era como constante las correcciones como } \\
\text { con un paso atrás" }\end{array}$ & \\
\hline
\end{tabular}


Tabla 4: (continuación)

\begin{tabular}{|c|c|c|}
\hline Ejes de Análisis & Fragmentos claves & $\begin{array}{l}\text { Síntesis interpretativa, } \\
\text { conceptualizaciones relevantes }\end{array}$ \\
\hline \multirow[t]{5}{*}{ Procedimental } & $\begin{array}{l}\text { Párrafo 9: "en la teoría, porque en urbanismo uno, dos ellos } \\
\text { te dicen que existe como este análisis de la ciudad de Kevin } \\
\text { Lynch y como ciertos análisis que son espaciales, } \\
\text { morfológicos, etc. para poder después empezar a diseñar } \\
\text { como a otra escala y ese cambio }\end{array}$ & $\begin{array}{l}\text { El "rayado de cancha" de qué tipos } \\
\text { de planos se requerían sirvieron } \\
\text { para clarificar. }\end{array}$ \\
\hline & $\begin{array}{l}\text { Párrafo 10: "fue bueno tener el rayado de cancha de la } \\
\text { primera semana cuando ustedes enviaron esa... ese formato } \\
\text { que decía los tipos de planos y que es lo que se busca } \\
\text { obtener por cada uno, yo creo que igual fue bueno tener } \\
\text { como esa base para arrancar el taller. Yo creo que ha servido } \\
\text { harto que... tampoco estemos como disparando con los ojos } \\
\text { cerrados. }\end{array}$ & $\begin{array}{l}\text { El establecer un FODA nos hace } \\
\text { mucho más fácil la tarea por } \\
\text { ejemplo, lo que nos toca a nosotras } \\
\text { ahora en Valdivia: enfocarte todo el } \\
\text { tiempo en el humedal y en fondo no } \\
\text { resuelves una problemática que } \\
\text { puede tener la población no se... } \\
\text { que está al lado y que debería darles } \\
\text { una solución también como } \\
\text { habitacional o todo. }\end{array}$ \\
\hline & $\begin{array}{l}\text { Párrafo 11: "que encuentro que se nos hace mucho más fácil } \\
\text { la tarea cuando establecemos un FODA que ya está } \\
\text { intencionado. }\end{array}$ & \\
\hline & $\begin{array}{l}\text { Párrafo 12: "estamos hablando de un contexto, estamos } \\
\text { hablando todo un... una sociedad y que al analizar y } \\
\text { proponer un proyecto urbano tampoco podemos ser } \\
\text { sesgados, por ejemplo, lo que nos toca a nosotras ahora en } \\
\text { Valdivia: enfocarte todo el tiempo en el humedal y en fondo } \\
\text { no resuelves una problemática que puede tener la población } \\
\text { no se... que está al lado y que debería darles una solución } \\
\text { también como habitacional o todo" }\end{array}$ & \\
\hline & $\begin{array}{l}\text { Párrafo 13: "la diferencia que hay entre todas las... todos los } \\
\text { ramos de urbanismo que tuvimos y este es que, por lo menos } \\
\text { a mí, fue primera vez que nos dijeron...eh.. "esto también } \\
\text { tiene que ver con el espacio", no es con, no son solamente } \\
\text { la formas de las manzanas, ni como están compuestas, ni } \\
\text { cómo se diseñó, no se po, París, Barcelona... si no que } \\
\text { entiendan esto de manera espacial y como está compuesto } \\
\text { a través de esto y esto otro, o sea, ese, el hecho de haber, } \\
\text { como, desmenuzado cada una de las partes... y hacer una } \\
\text { análisis individual y después juntar todo, eh, para mí me hizo } \\
\text { como ordenar, un poco... la, la cabeza... y como poder } \\
\text { llegar, y ahora, para mi es mucho más sencillo poder analizar } \\
\text { cualquier pieza urbana, no sé si una ciudad completa o no, } \\
\text { puede ser, pero si, si me dicen analiza este trozo ya, sé que } \\
\text { hacer y sé que dimensiones puedo tomar" }\end{array}$ & \\
\hline Actitudinal & Responsabilidad y/o del arquitecto en la ciudad & del arquitecto en la ciudad. \\
\hline
\end{tabular}

A nivel particular, en la primera aplicación del instrumento que contempla conceptualizaciones básicas los niveles son el nivel 3, nivel 4 y nivel 5. Esto implica que el nivel de transferencia del punto de vista del aprendizaje permite movilizar conocimientos para explicar, proyectar y ejemplificar a otros. Mientras que en la tercera fase de aplicación del instrumento se aprecian dos niveles que no están presenten en los anteriores resultados y que son el nivel 1 y el nivel 2. Esto implica que los aspectos procedimentales tienen mayor complejidad cognitiva a evaluar metacognitivamente. Resulta llamativo en los resultados que el tercer instrumento KPSI los resultados no sean los esperados, teniendo en cuenta que es el momento en el que, tras el proceso metodológico guiado en el que se aplican las herramientas urbanas y se desarrollan estrategias más novedosas para el alumno, resulta que en la fase en la que tiene que acometer el proceso creativo para el que se ha venido preparando durante los cuatro años anteriores sea en el que encuentre más dificultad. En cuanto al grupo focal, resulta interesante destacar que todas las apreciaciones realizadas por los participantes, son descubrimientos que ellos realizaron en el momento de la conversación grupal y, en su mayoría, descubren en ese momento la relevancia para su perfil curricular del aprendizaje realizado a través de la metodología desarrollada en el Taller $\vee$ y del aprendizaje basado en problemas, en este caso, el borde costero de Penco (Chile). 


\section{CONCLUSIONES}

Se puede concluir que el uso de los instrumentos de metacognición como estrategia complementaria a la metodología asentada en la UBB, permitió orientar la didáctica de Taller V para dirigir mejores resultados en la comprensión de los procesos de aprendizaje. Gracias a ello, las profesoras pudieron evaluar fortalezas y debilidades del proceso e ir ajustando el método para fortalecer y consolidar los saberes conscientes de los alumnos para enfrentar nuevos desafíos académicos. Por lo tanto, la incorporación de instrumentos de metacognición en el Taller $\mathrm{V}$ permitió robustecer lo aprendido y, principalmente, que los alumnos tomaran conciencia de ello. Complementariamente, fue posible detectar que los estudiantes no están acostumbrados al ejercicio metacognitivo.

En el proceso de resolución del problema propuesto realizan las tareas sin evidenciar o hacer consciente qué elementos les falta, qué conceptos les cuesta más adquirir o en para qué competencias están mejor preparados. Por ello, es necesario que los instrumentos de metacognición sean incorporados e implementados desde primeros talleres de formación en la carrera de arquitectura. De esta forma, los alumnos enfrentarían de mejor forma el Taller $\mathrm{V}$ de Diseño Urbano que se plantea como asignatura integradora de materias prácticas y teóricas desarrolladas en el área de urbanismo. Finalmente, es necesario destacar que la metacognición resulta ser un instrumento útil y complementario en la metodología de aprendizaje basada en problemas y en los procesos creativos en los que los resultados, bien concretos o abstractos, no exponen o relevan todos los aprendizajes y competencias adquiridos en su proceso.

\section{REFERENCIAS}

Abusaada, H.G. y A. Elshater, Knowledge-Based Urban Design in the Architectural Academic Field. In Knowledge-Based Urban Development in the Middle East, 204-227, IGI Global, Hershey, USA (2018)

Arze, G., C. Mawromatis, F. Corvalán y H. Orozco, El Diseño Urbano como experiencia docente: Estrategias y oportunidades de una formación temprana, Revista de Urbanismo, 14(27), 2 (2012)

Atman, C., M.E. Cardella, J. Turns y R. Adams, Comparing freshman and senior engineering design processes: an indepth follow-up study, doi: 10.1016/J.DESTUD.2004.09.005, Design Studies, 26(4), 325-357 (2005)

Bassanese, S., B. Rodeghiero y A. Espanyol, Pursuing Resilience in Architectural Design through International Experimental Projects: Exploring New Boundaries in the Design Studio Pedagogy, In Transdisciplinary Urbanism and Culture, 23-39, Springer, Cham (2018)

Burón, J., Enseñar a aprender: Introducción a la metacognición, Mensajero, Bilbao, España (1990)

Coll, C., E. Martín y otros 5 autores, El constructivismo en el aula, Graó, Barcelona, España (1997)

Cross, N., The nature and nurture of design ability, doi:10.1016/0142-694X(90)90002-T, Design Studies, 11(3), 127-140, (1990)

Dewey, J., P.A. Schilpp y L.E. Hahn (Eds.), The Philosophy of John Dewey, 2a Ed., Tudor, Nueva York, USA (1951)

Diamond A. y K. Lee, Interventions shown to Aid Executive Function Development in Children 4-12 Years Old, Science, 19, 959-964 (2011)

Donovan, E., Sustainable Architecture Theory in Education: How Architecture Students Engage and Process Knowledge of Sustainable Architecture. In Implementing Sustainability in the Curriculum of Universities, 31-47, Springer, Cham (2018)

Flavell, J.H., Metacognitive aspects of problem solving. In The nature of intelligence, 231-235, John Wiley \& Sons, Halsted Press, Nueva York, USA (1976)

Flores-Lázaro, J.C., R.E. Castillo-Preciado y N.A. Jiménez-Miramonte, Desarrollo de funciones ejecutivas, de la niñez a la juventud, doi: 10.6018/analesps.30.2.155471, Anales de Psicología, 30(2), 463-473 (2014)

Glaser, R., Learning theory and instruction, In International perspectives on psychological science, II: The State of the Art, Lawrence Erlbaum Associates, Hove, UK (1994)

Gomez-Galan, J., Educational Architecture and Emerging Technologies: Innovative Classroom Models, Revista Educativa Hekademoss, 22, 7-18 (2018)

Hargrove, R.A., Assessing the long-term impact of a metacognitive approach to creative skill development, doi: 10.1007/s10798-011-9200-6, International Journal of Technology and Design Education, 23(3), $489-517$ (2013)

Hernández, S., El modelo constructivista con las nuevas tecnologías: aplicado en el proceso de aprendizaje, Revista de Universidad y Sociedad del Conocimiento, 5(2), 26-35 (2008)

Icarte, G.A. y H.A. Labate, Metodología para la revisión y actuación de un diseño curricular de una carrera universitaria incorporando conceptos de aprendizaje basado en compentencias, Formación Universitaria 9(2), 3-16 (2016)

Lagos, R., Cambios en la Formación Profesional, Revista CA, 124, 60-61 (2005)

Lazo, L., Estrategia para la enseñanza y el aprendizaje de la Química General para estudiantes de primer año de Universidad, Revista Electrónica Diálogos Educativos, 23(12), 66-89 (2012) 
Mabardi, J.F., Maestría del Proyecto: Apuntes para la Práctica de la Enseñanza del Proyecto, 1a Ed., Universidad del Bío Bío, Concepción, Chile (2012)

Morales, P. y V. Landa, Aprendizaje Basado en Problemas, doi:0.4067/S0718-50062012000500003, Theoria, 13(1), 145157 (2004)

Muñoz, M.J., Proyectar/Investigar: Método de educación dialógica, aplicado al desarrollo de competencias, en la iniciación al diseño sostenible, Formación Universitaria, 2(2), 17-26 (2009)

Mutaqi, A.S., Architecture Studio Learning: Strategy to Achieve Architects Competence, doi: 10.1051/shsconf/20184104004, SHS Web of Conferences, 41, 04004 (2018)

Nelson, T.O., New Theory and Data on Metacognitive Monitoring and Control in Different Contexts and by Different Individuals. In Metacognition: Process, Function and Use, Kluwer, Boston, USA (2002)

Osses, S. y S. Jaramillo, Metacognición: un camino para aprender a aprender, doi: 10.4067/S0718-07052008000100011, Estudios Pedagógicos, 34(1), 187-197 (2008)

Raposo, J., Dibujar, procesar, comunicar: el proyectar arquitectónico como origen de un proceso grafico-plástico. Implicaciones docentes, doi:10.4995/ega.2014.3091, EGA. Revista de Expresión Gráfica Arquitectónica, 19(24), 92, (2014)

Sawyer, R.K., Teaching and Learning How to Create in Schools of Art and Design, Journal of the Learning Sciences, 27(1), 137-181 (2018)

Schön, D.A., Knowing-in-action: The new scholarship requires a new epistemology, Change: The Magazine of Higher Learning, 27(6), 27-34 (1995)

Voyatzaki, M. y D. Gourdoukis, From Morphology to Morphogenesis: On Speculative Architectural Design Pedagogy, In Handbook of Research on Form and Morphogenesis in Modern Architectural Contexts, 20-40, IGI Global, Hershey, USA (2018)

Young, D.B. y P. Tamir, Finding out what Students know, The Science Teacher, 44, 27- 28 (1977) 